\title{
Spectral state transitions in low-mass X-ray binaries - the effect of hard and soft irradiation
}

\author{
B. F. Liu ${ }^{1,2}$, F. Meyer ${ }^{2}$, and E. Meyer-Hofmeister ${ }^{2}$ \\ 1 National Astronomical Observatories/Yunnan Observatory, Chinese Academy of Sciences, PO Box 110, Kunming 650011, PR China \\ 2 Max-Planck-Institut für Astrophysik, Karl-Schwarzschildstr. 1, 85740 Garching, Germany \\ e-mail: emm@mpa-garching.mpg.de
}

Received 7 April 2005 / Accepted 24 May 2005

\begin{abstract}
In neutron star and black hole X-ray binaries the transitions between the two spectral states, hard and soft, signal the change between accretion via a hot advection-dominated flow(ADAF) and disk accretion. In a few cases the hard/soft transition was observed during the rise to the nova outburst, but in most cases only the soft/hard transition during the luminosity decrease. Surprisingly the luminosities at the second transition are always lower by a factor of 3 to 5 . A model for this hysteresis was presented in a preceding paper where it was shown that this switch in the accretion mode at different mass accretion rates and therefore different luminosities is caused by the different amount of Compton cooling or heating of the accretion disk corona as it is irradiated by hard or soft radiation from the central light source, respectively. We now give detailed results for the dependence on the hardness of this radiation and the radiation efficiency. We further discuss the influence of the inclination and a possible warping of the disk on the observed hysteresis.
\end{abstract}

Key words. accretion, accretion disks - black hole physics - X-rays: binaries - stars: neutron stars: individual: Aq1 X-1, GX339-4, XTE J1550-564, XTE J1650-500

\section{Introduction}

Of great interest in black hole physics are the properties of the matter flow towards a compact object. Viscous differentially rotating gas flows determine the accretion processes around black holes, where viscous transport of angular momentum outward allows the gas to spiral inward towards the compact object. For a detailed discussion of the different possible modes of accretion see the work by Chen et al. (1995) and the review by Narayan et al. (1998).

The difference in observed spectra arising from the innermost accretion flow, can be used to study these accretion modes, which are the same in stellar black holes and supermassive black holes in the centers of galaxies. Applications provide information for truncated thin disks in stellar mass systems, as e.g. the black hole binary nova Muscae 1991 (Esin et al. 1997), as well as in low luminosity active galactic nuclei, e.g. M 81 (Quataert et al. 1999). A rich documentation is present for accretion in low-mass X-ray binaries containing a black hole or a neutron star primary (Chen et al. 1997; McClintock \& Remillard 2005). The observations display the spectra of the X-ray radiation for different modes of accretion and correspondingly different spectral states: (1) a very hard spectrum (up to $100 \mathrm{keV}$ ) originating from the very energetic particles of a hot spherical advection-dominated flow (ADAFtype), (2) a soft spectrum (a few keV) radiated from the much cooler geometrically thin accretion disk. This is a simplified picture. In addition to the mentioned X-ray states of low and high luminosity also an "intermediate" state and a "very high" state can be found. McClintock \& Remillard (2005) present an overview of the emission states of black-hole binaries where the combined X-ray and multi-frequency spectral characteristics of the sources are taken into account and a clearer definition of state names is suggested.

An interesting documentation of spectral changes are the transitions between hard and soft state observed for lowmass X-ray binaries, both neutron star and black hole systems (Tanaka \& Shibazaki 1996), and also for high-mass X-ray binaries. The first observed change for a neutron star was found by Mitsuda et al. (1989) for 1608-522, and for a black hole system by Ebisawa et al. (1994) for GRS 1124-684, Nova Muscae 1991. A first modeling of the spectra was presented by Esin et al. (1997) using the concept of an inner advection-dominated accretion flow. Up to now the number of observations and the quality of spectra has very much increased, so that these data allow us to learn from the compariog theoretical models with observations.

There is one special feature in the appearance of the transition of spectral states: considering an X-ray nova outburst light curve, we see that the hard-soft transition does not occur at the same luminosity as the soft-hard transition, which occurs at a luminosity lower by a factor of about 5. In recent work 
(Meyer-Hofmeister et al. 2005, hereafter MLM05) this surprising hysteresis could be explained as arising from the different amount of Compton cooling or heating acting on the accretion disk corona at the time of the state transition. During phases of low mass flow in the disk, as in quiescence and the early rise to outburst, the inner disk region is filled with an ADAF, the radiation is very hard, and the hot photons lead mainly to Compton heating of the corona. If otherwise during the time around outburst maximum the mass flow rate in the disk is high, the thin disk reaches inward to the last stable orbit, the radiation is soft and the Compton effect acts to cool the corona. This difference in irradiation leads to a different vertical structure of the corona and a different amount of evaporation of gas from the disk.

In Sect. 2 we summarize the observations for hysteresis. In Sect. 3 we briefly describe the physics of our model for the interaction of disk and corona and the evaporation of gas from the cool disk. Computating the vertical structure of the corona allows us to study the Compton effect on the corona in detail and to evaluate the dependence on the hardness of the hard radiation. In Sect. 4 the results of the model calculations are shown. Since the irradiation from the inner region affects the evaporation rate, the accretion rate and efficiency of radiation enter into the results. We compare these results with observations in Sect. 5. The intermediate state between hard and soft spectral state is discussed in Sect. 6, further we mention a possible effect of the inclination under which we observe a binary system in connection with flaring or warping of the disk. The conclusions follow in Sect. 8.

\section{Observed hysteresis in lightcurves of X-ray binaries}

Data for spectral state transitions in individual sources were already discussed in the first paper on hysteresis (MLM05). In Table 1 we summarize data on hysteresis now available in the literature. The values for the hysteresis given there are approximate values, either derived in the work referenced or deduced for the present paper from light curves and hardness ratios HR2 given in the literature (state transitions taken as occurring when HR2 $=(5-12 \mathrm{keV}) /(3-5 \mathrm{keV})=1.5$, McClintock \& Remillard 2005). The existence of a hysteresis is well-documented in the hardness-intensity diagrams, but it is difficult to deduce the ratio between the luminosities at the transitions. The hardness used in these diagrams is different for different observations and it is difficult to deduce the value of hysteresis from this information. To deduce a reliable value for the hysteresis is also difficult due to the fact that the time of transition is not clearly documented, since the hard/soft and, even more, the soft/hard transition take some time (as discussed in following sections). This means the values in Table 1 only show a range. We also refer to the description of spectral states in the review of McClintock \& Remillard (2005, Sect. 4.3): the low/hard (LH) and high/soft (HS) state correspond to our nomenclature hard and soft.

Historically the hysteresis effect was first pointed out by Mijamoto et al. (1995). A first detailed analysis of hysteresis in transient low-mass X-ray binaries was performed by Maccarone \& Coppi (2003a), who discussed the observations of Aql X-1 during the 1999 outburst in detail, which is one of the best examples, with two spectral transitions clearly documented at different count rates. For data and references of further interesting sources, see Table 1 . The source 4U 1543-47 (Park et al. 2004) was included because it has a classical X-ray nova outburst light curve. McClintock \& Remillard (2005) show a hard phase in the very beginning in their Fig. 4.2. Since the luminosity at outburst maximum is in the range of the Eddington luminosity, the luminosity at the hard/soft transition (expected at a few percent of this value) cannot be extracted from the data. Meanwhile the transition soft/hard is well documented.

\subsection{The hardness of observed spectra}

Since the hardness of the radiation from the inner region has an essential influence on the evaporation process, the hardness at the time of the change from hard to soft state is of interest. But only a limited number of hard spectra are available for the sources listed in Table 1.

Maccarone \& Coppi (2003b) analyzed data from the May/June outburst 1999 of Aql X-1 and present a spectrum taken shortly before transition to the soft state. This spectrum ideally documents the irradiation at the state transition. We evaluated the mean photon energy as about $h \bar{v} \approx 90 \mathrm{keV}$. The true value might be higher depending on the poorly known spectrum at $\geq 100 \mathrm{keV}$. In the work by Muno et al. (2002, Fig. 1) hard and soft colors for different sources are shown. The hard color of $4 \mathrm{U} 1705-440$ at the hard/soft state transition seems comparable or slightly less than that of Aql X-1.

Wardziński et al. (2002) analyzed hard spectra of GX 339-4 taken by the soft $\gamma$-ray OSSE detector on board CGRO, simultaneous with Ginga and RXTE observations. The energy range reaches up to several hundred $\mathrm{keV}$. The source flux at the time the spectra were taken was about 1 percent of the Eddington luminosity, therefore close to a value where we expect the state transition (see further discussion of these spectra by Zdziarski et al. 1998; Zdziarski \& Gierliński 2004). Evaluation of the spectrum from 1997 leads to $h \bar{v} \approx 100 \mathrm{keV}$. The large errors at high energies cause quite an uncertainty in the result.

For XTE J1550-664 a hard spectrum (PCA + HEXTE data, $18-200 \mathrm{keV}$ ) taken 16 days before the hard/soft transition of the outburst in 2000 is shown by Rodriguez et al. (2003). We evaluate about $90 \mathrm{keV}$ for the mean photon energy, assuming no significant contributions at higher energy. An averaged hard spectrum taken during the 2000 outburst (Arefiev et al. 2004) is less hard.

Rossi et al. (2004) present a study of the 2001/2002 outburst of the transient source XTE J1650-500. The beginning of the outburst was well covered, but the hard/soft transition discussed there could already be the transition to the very high state. A spectrum taken right before this transition (Rossi, private communication 2005) is not as hard as the spectra from other sources discussed above. 
Table 1. Observations of X-ray transients

\begin{tabular}{|c|c|c|c|c|c|}
\hline $\begin{array}{l}\text { Source } \\
\text { name }\end{array}$ & Class & Year & $\begin{array}{l}\text { Flux ratio: } \\
\text { hysteresis }\end{array}$ & $\mathrm{keV}$ band & References \\
\hline \multirow[t]{2}{*}{ Aql X-1 } & ns & 1999 & 7 & RXTE/PCA, 1-12 keV & Maccarone \& Coppi (2003a) \\
\hline & & 2000 & $10 ?$ & RXTE/PCA, 2-60 keV & Maitra \& Bailyn (2004) \\
\hline $4 \mathrm{U} 1705-440$ & ns & 1999 & 4 & RXTE/ASM, 2-12 keV & Muno et al. (2002) \\
\hline \multirow[t]{3}{*}{ GX 339-4 } & bh & 1998 & 3.5 & RXTE/ASM, 3-12 keV & Zdziarski \& Gierliński (2004) \\
\hline & & 2002 & 10. & RXTE/ASM, 3-12 keV & Zdziarski \& Gierliński (2004) \\
\hline & & & HID & RXTE/PCA, 3-21 keV & Homan \& Belloni (2005) \\
\hline \multirow[t]{3}{*}{ 4U 1543-475 } & bh & 2002 & small? & RXTE/ASM, 2-12 keV & McClintock \& Remillard (2005) \\
\hline & & & & RXTE/PCA, 3-25 keV & Park et al. (2004) \\
\hline & & & & RXTE/PCA+HEXTE, 3-200 keV & Kalemci et al. (2005) \\
\hline \multirow[t]{3}{*}{ XTE J1550-564 } & bh & 1998 & around $\geq 10^{*}$ & RXTE/ASM, 1-12 keV & Kubota \& Done (2004) \\
\hline & & 2000 & $2^{* *}$ & RXTE/CA+HEXTE 2-200 & Rodriguez et al. (2003) \\
\hline & & 2000 & HID & RXTE/ASM, 1-12 keV & Maccarone \& Coppi (2003a) \\
\hline \multirow[t]{3}{*}{ XTE J1650-500 } & bh & 2001 & $5-10$ & RXTE/PCA+HEXTE, 3-150 keV & Rossi et al. (2004) \\
\hline & & & HID & RXTE/PCA + HEXTE, 3-200 keV & Corbel et al. (2004) \\
\hline & & & HID & RXTE/PCA, 3-21 keV & Homan \& Belloni (2005) \\
\hline GRS 1739-278 & bh & 1996 & 5 & RXTE/ASM, 2-12 keV & McClintock \& Remillard (2005), Borozdin et al. (1998) \\
\hline H1743-322 & bh & 2003 & HID & RXTE/PCA, 3-21 keV & Homan \& Belloni (2005) \\
\hline \multirow[t]{2}{*}{ GRS 1748-288 } & bh & 1998 & HID & RXTE/PCA, 1-12 keV & Maccarone \& Coppi (2003a) \\
\hline & & & about 10 & IRS-P3/IXAE, 2-18 keV & Naik et al. (2000), McClintock \& Remillard (2005) \\
\hline \multirow[t]{2}{*}{ XTE J1859+226 } & bh & 1999 & HID & RXTE/ASM, 1-12 keV & Maccarone \& Coppi (2003a) \\
\hline & & & HID & RXTE/PCA, 3-21 keV & Homan \& Belloni (2005) \\
\hline XTE J2012-381 & bh & 1999 & HID & RXTE/ASM, 1-12 keV & Maccarone \& Coppi (2003a) \\
\hline
\end{tabular}

Notes: The sources listed as black holes in the class column are confirmed black holes or black hole candidates. The values given for the hysteresis in the table are approximate values (see text). ${ }^{*}$ Fast rise from low/hard to very high state, no reliable luminosity value for the hysteresis. ${ }^{* *}$ Transition from low state to intermediate state.

HID: a hardness-intensity diagram shows the hysteresis for these systems (different energy bands, difficult to derive a hysteresis value). RXTE $=$ Rossi X-ray Timing Explorer with ASM = All Sky Monitor and PCA $=$ Proportional Counter Array. IRS-P3/IXAE = IRS -P3 satellite at the Indian X-ray Astronomy Experiment.

\section{The spectral state transitions}

\subsection{The general picture}

To analyze of the spectral state transitions in low-mass X-ray binaries we take the commonly accepted picture for the two accretion modes: (1) accretion via an optically thick geometrically thin cool disk (cool compared to a coronal flow) or (2) an optically thin spherically extended hot flow, ADAF-type two-temperature solution. Usually at larger distances from the compact object, the accretion flow always is of the first type mentioned, while closer to the central object both types are possible. The radiation is dominated by the flow in the innermost region; that is, a soft spectrum arises from disk accretion, and a hard spectrum from the hot flow of energetic particles. X-ray novae are good candidates for studying changes between the two modes. A hard spectrum in quiescence during mass accumulation in the disk and a soft one after a dwarf-nova type instability had triggered an outburst (Meyer-Hofmeister \& Meyer 1999). The importance of irradiation had already been pointed out by de Kool \& Wrickramasinghe (1999).
Caused by the interaction of disk and corona, gas evaporates from the disk into the corona and flows in the form of a hot advection-dominated flow, whereby the mass flow rate in the thin disk is diminished. The evaporation rate increases with decreasing distance from the compact object, but reaches a maximum at a certain distance. This maximum rate determines the switch between the accretion modes in the inner region. Only if the mass flow in the outer disk is larger than this value will the disk "survive" this reduction in mass flow and continue inward. Otherwise the disk becomes truncated at a certain distance depending on the mass flow rate, and from then on, all mass flows inward via the coronal/ADAF flow. This truncation of the inner disk during phases of low mass flow rate has long been recognized as an essential feature of disk evolution (Mineshige et al. 1998), which also appeared in numerical simulations (Cannizzo 1998, 2000; Dubus et al. 2001). A recent systematic analysis by Done \& Gierliński (2004) using all data available from galactic binary systems on changes of spectra and truncation radii as a function of the accretion rate confirms this picture. 


\subsection{The effect of irradiation on state transitions}

In the work by Meyer et al. (2000) the interaction of disk and corona was approximated by a one-zone model incorporating the standard equations of viscous hydrodynamics (see also Liu et al. 2002). As became clear in the recent work on hysteresis (MLM05), Compton cooling and heating by radiation from the innermost region acting on the vertically extended corona is an important process. The hysteresis is mainly caused by Compton cooling in the soft state, but irradiation in the hard state also plays an important role.

Evaporation has three important features: (a) the rates increase towards smaller distance $R$, (b) the rates have a maximum value at about several hundred Schwarzschild radii, and (c) from that distance on inward the coupling between electrons and ions becomes poor. Hard irradiation leads to higher mass evaporation rates, a higher maximal value, and therefore the spectral state transition at a higher luminosity than in the case of soft irradiation (compare Fig. 1 in MLM05). The harder the hard radiation is, the higher the evaporation rate at maximum. Our recent computations were based on irradiation as hard as $100 \mathrm{keV}$. Since the spectra observed for X-ray transients show quite a difference in hardness, we take this into account for the evaluation of evaporation rates. As described in the foregoing paper (Sect. 4.3), the Compton cooling/heating rate per unit volume taken is the sum of Compton cooling and heating (inverse Compton and Compton effect):

$q_{\text {Comp }}=\frac{4 k T_{\mathrm{e}}-h \bar{v}}{m_{\mathrm{e}} c^{2}} n_{\mathrm{e}} \sigma_{\mathrm{T}} c u$,

with $k$ the Boltzmann constant, $T_{\mathrm{e}}$ electron temperature, $m_{\mathrm{e}}$ electron mass, $c$ velocity of light, $n_{\mathrm{e}}$ electron particle density, $\sigma_{\mathrm{T}}$ Thomson cross section, and $u$ the energy density of the photon field. At transition the dominant contribution comes from photons from the central source, so those from the disk underneath can be neglected here. Also Compton cooling by photons of the secondary stars is generally negligible.

For the flux from the central region in the soft state, we now take a slightly different form compared to that in MLM05. We replace $H / R$ ( $H$ scaleheight $)$ by the term $2 \cos \delta=2 z /\left(r^{2}+\right.$ $\left.z^{2}\right)^{1 / 2}$, where $\delta$ is the inclination angle under which the inner disk appears at height $z$. The formulae then is

$$
F=\frac{L}{4 \pi R^{2}} \frac{2 z}{\left(r^{2}+z^{2}\right)^{1 / 2}},
$$

where $L$ is the luminosity of the central source, which is related to the central mass accretion rate $\dot{M}$ as $L=\eta \dot{M} c^{2}$. The more detailed formula results in a less strong Compton effect than the expression used earlier and therefore a higher maximal evaporation rate.

\section{Model calculations for hysteresis}

In our computations we took a black hole mass $M=6 M_{\odot}$; the results can be scaled for other masses, and we show the results as measured in Eddington accretion rate $\dot{M}_{\text {Edd }}=L_{\text {Edd }} / 0.1 c^{2}$ with $L_{\text {Edd }}=4 \pi G M c / \kappa, \kappa$ electron scattering opacity, and in Schwarzschild radius $R_{\mathrm{S}}=2 G M / c^{2}$. The viscosity has a strong influence on the results, as shown in earlier work (Meyer-Hofmeister \& Meyer 2001; Liu et al. 2002) and also pointed out by Różańska \& Cerny (2000). We use the value $\alpha=0.3$ supported by modeling of X-ray binary spectra (Esin et al. 1997), and also application to accretion disk evolution (Meyer-Hofmeister \& Meyer 1999).

\subsection{Evaporation rates - dependence on hardness of irradiation and radiation efficiency}

We determined the maximal evaporation rates (the rate determining the state transition) in the hard state for 30 to $120 \mathrm{keV}$ mean photon energy of the radiation from the central source. The peak value has to be consistent with the accretion rate taken for the irradiation. For this consistency the efficiency $\eta$ with which radiation is produced from accretion enters. In the recent paper we had taken the value 0.05 for the efficiency in the hard state. In Fig. 1 we show the earlier results for $100 \mathrm{keV}$ mean photon energy as dashed lines to illustrate the procedure for finding the peak evaporation rate for the given efficiency $\eta=0.05$ or 0.025 , marked by filled or open dots. To determine each dot, a series of evaporation rates at different distances $R$ from the center had to be evaluated to find the maximum, and the maximum had to be consistent with respect to the radiation produced in the central region. With this procedure we determined the maximal evaporation rates for different hardness and efficiency. The rates for $\eta=0.05$ and 0.025 are marked by filled and open dots. Along each solid curve the efficiency decreases towards zero without irradiation (square).

The cross marks the peak evaporation rate for soft irradiation when the disk reaches inward to the last stable orbit. This is the case at the soft/hard transition. The difference between this value and the peak evaporation rate for hard radiation yields the hysteresis value.

\subsection{Results for spectral state transitions}

The peak evaporation rates for given hardness and efficiency of radiation provide the full picture of accretion rates that correspond to the spectral transitions from hard to soft state. Taking, e.g., the radiation efficiency as 0.05 , the filled dots in Fig. 1 give the truncation radii expected at the time of change from ADAFtype accretion to thin disk accretion. These radii are larger than those without Compton effect.

\section{Comparison with observations}

\subsection{Hysteresis}

In our section on observations (Sect. 2) we already have pointed out that hysteresis is clearly observed for several sources, but it is difficult to deduce the ratio of the luminosities corresponding to the two transitions. There are several reasons for this. The hard/soft transition is often is not well- sampled and, the spectral change takes some time (days to weeks), so that the exact time of transition is an interpretation of the data. A further uncertainty enters since different energy bands are used for the observations. The hardness-intensity diagrams presented by 


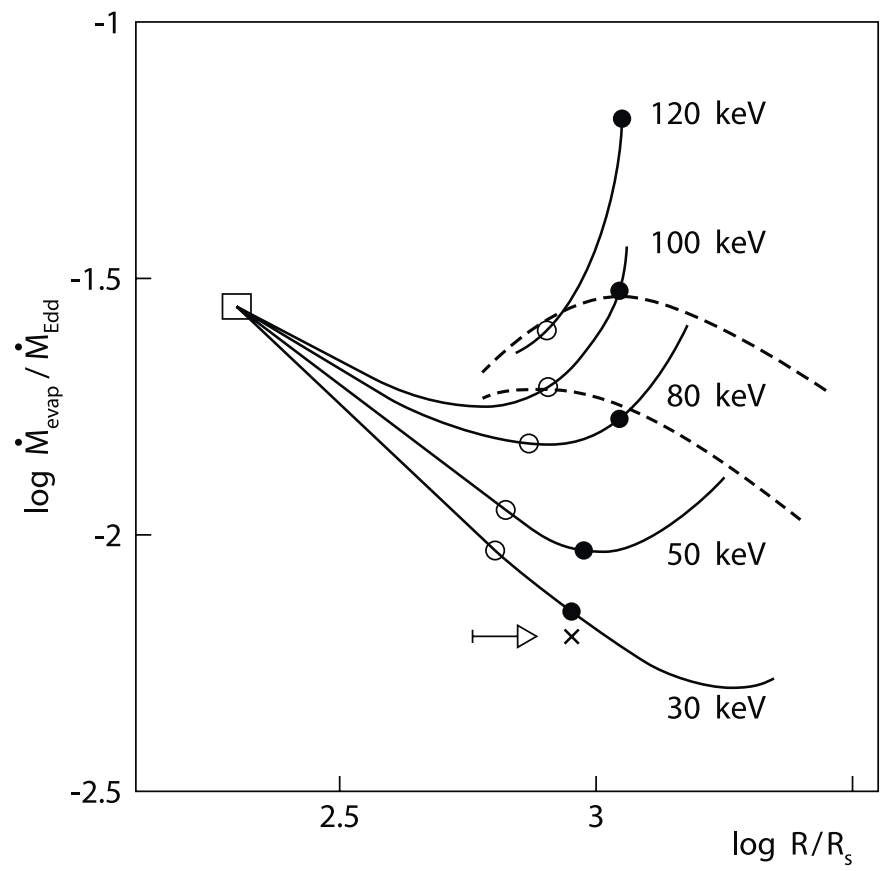

Fig. 1. Evaporation rates at the hard/soft spectral transition for different hardness of the radiation from the central region. Dashed curves: evaporation rates for mean photon energy $h \bar{v}=100 \mathrm{keV}$, upper curve radiation efficiency $\eta=0.05$, lower curve 0.025 , dots mark maxima. Solid curves: maxima of evaporation rates for $h \bar{v}=30-120 \mathrm{keV}$. Along each curve, efficiency of radiation decreases from right to left in the diagram, values $\eta=0.05$ and 0.025 marked by filled and open dots. The point where the curves converge (square) corresponds to $\eta=0$, that is, no irradiation. All rates are given in units of Eddington accretion rate, drawn at the truncation radius $R$ (in units of Schwarzschild radius $R_{\mathrm{S}}$ ). The hysteresis follows as the ratio between the peak evaporation rate in the hard state and that in the soft state (cross).

Corbel et al. (2004) and Homan \& Belloni (2005) show the effect of the different hardness bands used for the hardness ratios $(4.5-7.9) /(2.5-4.6) \mathrm{keV}$ and $(2-200) /(3-20) \mathrm{keV}$ respectively. The observationally deduced ratios of luminosities at transition, therefore, can only show a range of values.

In Table 1 for GX 339-4 two hysteresis values are listed, 3.5 for the 1998 outburst and 10 for the 2002 outburst. The soft/hard transitions both took place at $0.02 L_{\text {Edd }}$, a typical value, the hard/soft transitions at 0.07 (1998) and $0.2 L_{\text {Edd }}$ (in 2002), respectively (Zdziarski \& Gierliński 2004). How could such a difference arise? In our picture of state transition, this always should occur at the same luminosity and should not depend on the history as argued by Zdziarski \& Gierliński (2004). A possible explanation could be that the hard/soft transition at $0.2 L_{\text {Edd }}$ was already a change to the very high state and the then earlier transition from the low/hard to the soft state had occurred at a lower luminosity before the observations began (comparable to the situation in XTE J1650, see Sect. 2.1).

Our model calculations yield a clear dependence on hardness of the irradiation. In Fig. 2 we show this dependence together with luminosity ratios for some sources. As discussed in Sect. 2.1 only a few spectra taken at the time of the hard/soft

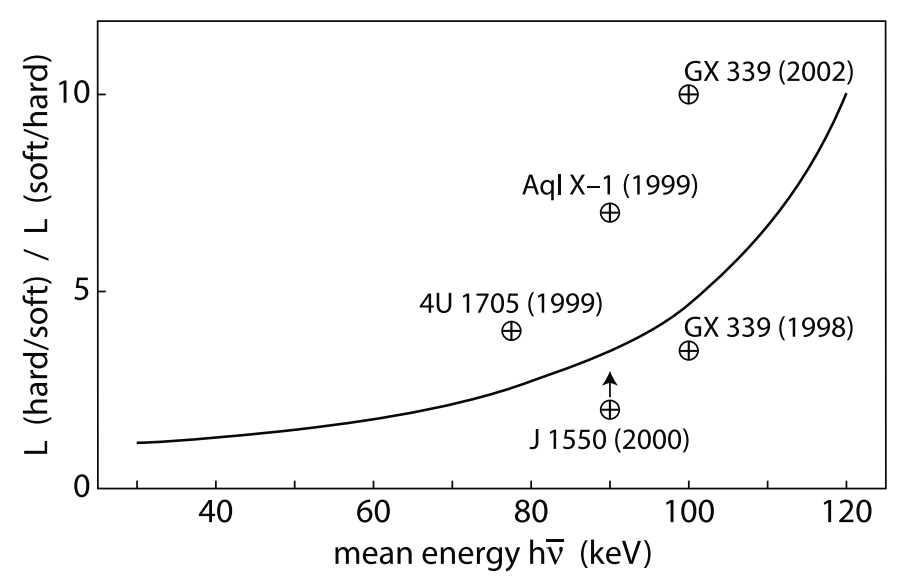

Fig. 2. Dependence of hysteresis on mean photon energy of irradiation. Model (solid line) and observed values for various sources (outburst year in parenthesis). For the difficulty to derive these values, see Sect. 5 .

transition are available. The results in Fig. 2 show the wide scatter. The hardness might be underestimated if radiation at higher energy bands, not included, were present. Recent work by Ling (2005) reports observations of the gamma-ray emitting source GRO J1719-24 at energy bands up to several hundred keV. Similarities with other black hole X-ray transients, GRO J0422+32, and with Cyg X-1 are mentioned, and it is not clear whether sources discussed here could also have X-ray emission in the gamma-ray energy region. Then a larger hysteresis would be expected.

\subsection{Truncation radii}

The fact that the state transitions occur at different luminosities leads to a dichotomy in the truncation radii for this luminosity interval where either state can be realized: the soft state in which the disk reaches to the last stable orbit, or the hard state in which the disk is truncated according to the massflow rate - radius relation. The theoretically expected truncation radii during outburst rise and decline are shown in Fig. 2 of MLM05. Probably related to the truncation radii are the variations in power density spectra as discussed by Olive et al. (2003) for $4 \mathrm{U}$ 1705-44.

If we compare our results for the truncation radii obtained from the Compton effect on the coronal structure taken into account (MLM05 and this work) we find radii larger than expected from observations. Originally, the one-zone model for evaporation without including the Compton effect yielded a disk truncation at a few hundred Schwarzschild radii when the spectral transition occurs (Meyer et al. 2000). The paper by Yuan \& Narayan (2004) compares the transition radii for several galaxies and galactic black hole sources. Note that sources in the hard state were also included, for which the disk truncation at spectral state transition should be less than or equal to the obtained value. The source XTE J1118 is a good example, as the disk edge moves inward from values of $10^{4}$ to $10^{3} R_{\mathrm{S}}$ during quiescence to about $100 R_{\mathrm{S}}$ during outburst, where the source remained in the hard state, as can occur in 
case of low accretion rates (Meyer-Hofmeister 2004). Using frequency-resolved spectroscopy of Cyg X-1 Gilfanov et al. (2000) estimated that the inner radius of the reflector, presumably an optically thick accretion disk, lies at about $100 R_{\mathrm{S}}$ in the hard spectral state. Based on observations of Nova Mus 1991, Zycki \& Done (1998) suggested that optically thick material within about $20-100 R_{\mathrm{S}}$ is generally present in the hard (low) state. The question arises whether a left-over inner disk is possible, together with the ADAF, a problem also connected with the intermediate state (see next section).

There is one parameter in our analysis which influences the truncation radii, heat conduction. In a forthcoming work we will evaluate the effect of a reduced heat conduction on the interaction of disk and corona. A further effect on the coronal structure and the location of the disk truncation might come from magnetic fields reaching into the corona.

\section{The intermediate state}

In the foregoing sections we discussed only two spectral states, hard and soft. The observations document an intermediate state which can persist for quite some time and sometimes does not even lead to a complete transition. Consistent with the explanation of the other two states, this state would correspond to an inner region with a comparable amount of mass flow accreting in the two forms via a hot extended corona and via a standard thin accretion disk. To understand how this can happen, a closer look at the coronal evaporation model is necessary. On top of a standard thin accretion disk a corona always forms in interaction with the disk, and this corona itself carries a part of the accretion flow. Analysis of the formation of the coronal flow/ADAF (Meyer-Hofmeister \& Meyer 2003) yielded how this coronal accretion flow increases with decreasing distance from the black hole until it reaches a maximum at a fixed distance between 100 and 1000 Schwarzschild radii. Inside of this distance either a pure coronal flow exists, if the disk has already become fully evaporated further outward, or the thin disk continues. What happens to the coronal flow above such a disk continuing inward after the hot flow has passed the distance where it reached its maximum? According to calculations a disk region farther in has a weaker corona and carries less coronal flow. Therefore the part that can no longer be carried inward must condense into the cool disk underneath (Liu et al. 2004).

This condensation process allows interesting aspects for the intermediate state, which we will discuss here and in a forthcoming work. Can all the former coronal flow condense so that the accretion in the innermost region occurs completely through the cool disk? This would mean that in the interior we have either a pure coronal flow in the hard state or a pure disk flow in the soft state with no room for the various forms of the observed intermediate state. We suggest a solution here for this apparent contradiction between the theoretical model and observations. This solution relies on the strong temperature dependence of the collisional coupling between the electrons and ions in the corona. For a cool corona, these two species are well coupled and efficiently exchange energy during the thermal timescale for heating and cooling of the corona. In this case frictional heat released in the ion component is readily transfered to the electrons and conducted to lower, denser levels and radiated away. This keeps the corona in a cool dense state as one goes to smaller radii, until it would formally disappear altogether yielding a perfect self consistent soft state in the interior. If, however, the coronal temperature is high in the beginning, coupling between electrons and ions is poor and the electrons cannot remove the frictional heat of the ions, which will then stay near virial temperatures. The farther one gets inward the hotter the ions become and the poorer the coupling between ions and electrons. This then will allow an intermediate state, in which a hot coronal flow passes above and below on both sides of a regular accretion disk with not much interaction between the two accretion streams.

Now it is very important that the regular coronal structure calculations just yield a beginning weakening of the coupling between electrons and ions at the distance from the black hole where the maximum coronal flow rate occurs; compare the shape of the hatched evaporation rates curve in Fig. 1. If the accretion rate is higher than this maximum, the disk does not fully evaporate and the coronal flow condenses effectively into the cool disk further in. If, however, the accretion rate drops to a value lower than the maximal possible coronal flow rate, the disk evaporates over some distance outside of the radius of the evaporation maximum and a gap is formed. From the outer border of the gap on through the whole gap region the accretion continues inward in the form of an ADAF. ADAF temperatures are nearly virial and are higher than those of coronae above a cool accretion disk at the same distance from the central black hole. Thus where the ADAF reaches the inner boundary of the gap i.e. the inner disk, it has a rather high temperature. This temperature is higher the farther in the gap extends, because the virial temperature increases with $1 / R$ as the radius $R$ decreases. The coupling becomes correspondingly poor between the electrons and ions, and the flow is therefore only partially able to cool off and to condense back into the disk. Though this partial condensation will feed the disk and keep it stretching all the way inward, another part of the accretion flow will stay in the hot near-virial flow above and below the disk and, on arriving in the interior, will give the hard spectral component to this intermediate state, the soft component being provided by the remaining disk flow.

If the accretion rate is quite close to the critical value (i.e. the maximal evaporation rate) or above it, the hard power law tail will only constitute a small fraction of the spectrum and, with the fluctuating accretion rate, will vary significantly. This would then account for the observed hard power law tail in the soft state.

\section{Discussion}

A number of factors can complicate a direct comparison between theoretical model and observations. In the soft state the luminosity is radiated from a thin innermost accretion disk. When this disk lies in the equatorial plane, the observer sees it foreshortened. The "observed" luminosity is then inclination dependent and must be corrected for this projection in order to obtain the true luminosity at the soft/hard transition. 
Further, should the inner disk be tilted out of the equatorial plane and precess, an additional and time-varying aspect of the accretion disk enters and would simulate an apparent variation of the transition luminosity, even when the true transition luminosity remains the same. Tilted and precessing accretion disks might be indicated by periodic shifts in color-intensity plots of low mass X-ray binaries.

Recently, Narayan \& McClintock (2005) investigated the effects of the inclination angles of black hole X-ray binary systems on their observational properties. Within their sample of 20 sources, they found none with an inclination angle larger than $75^{\circ}$ and suggested that this absence of eclipsing sources is due to a flaring of the accretion disk by about $15^{\circ}$, so that the disk permanently occults the X-ray source for the observer for all inclination angles that would allow us to see eclipses. Noisy light curves of sources with inclination between 70 and $75^{\circ}$ support this picture and indicate the presence of partial and time-varying coverage of the central source. Such a partial occultation could affect the determination of the luminosities at both hard/soft and soft/hard transitions.

A perhaps more remote possibility, which would however have a direct impact on the coronal evaporation mechanism itself and thus on the hysteresis, is a warping of the inner accretion disk. This would change the aspect under which the corona sees the irradiating innermost disk surface and thereby change the strength of the irradiation.

\section{Conclusions}

As one follows the light curve of an X-ray nova from early rise to late decline, observations show a profound change in the source spectrum from a hard to a soft one on the rise and back from a soft to hard one on the decline. These changes occur at characteristic luminosities, but remarkably, they are not the same for the two transitions. This hysteresis in the light curve could be understood as arising from the different type of irradiation coming from the innermost region, which is hard at the hard/soft transition but soft at the soft/hard transition. This difference leads to a different Compton cooling or heating of the coronal layers and results in a different coronal density and mass flow. The latter determines whether a disk can be truncated (hard state) or continues all the way to the black hole (soft state; see MLM05).

We have now carried out further analysis and shown how this hysteresis depends on the hardness of the irradiation. Unfortunately, only a few spectra taken at the moment of state transition are available. Also the contributions at the important high energies are uncertain. Thus the observational basis for a comparison is still fairly sparse. In addition, theoretical uncertainties arising from the rough "one-zone" modeling and the choice of its parameters together with the assumed planar inner disk geometry will enter into comparisons between observations and theoretical models.

Thus it is difficult to verify in detail the theoretically well understood dependence on the hardness of the hard irradiation in comparison with the data. It is also difficult to determine when and at which luminosity the transition actually happened. Different observations are taken in different energy bands. Since the change from one state to the other takes some time, this also clearly demands a more detailed understanding of the intermediate state between the hard and soft state. In our first discussion here we find a remarkable coincidence between the distance where the coronal flow rate reaches its maximum and that of the least thermal coupling between electrons and ions, which could perhaps explain the features of an intermediate state.

In conclusion one might take the explanation of the hysteresis as further strong support for the coronal evaporation model. It is surprising how this very simplified model appears to catch essential features of the accretion flows around compact objects. More observations of spectral state transitions in different systems, broad spectral energy coverage, and refinement of the theoretical modeling in the future might prove very fruitful.

Acknowledgements. One of the authors, B.F.L., thanks the Alexandervon-Humboldt Foundation for the award of a research fellowship during which this investigation started. We would like to thank Sabrina Rossi and collaborators for providing a spectrum of XTE J1650 at the time of transition during outburst rise.

\section{References}

Arefiev, V. A., Revnivtsev, M. G., \& Lutovinov, A. A. 2004, Astron. Lett., in print [arXiv:astro-ph/0404460]

Borozdin, K. N., Revnivtsev, M. G., Trudolyubov, S. P., et al. 1998, Astron. Lett., 24, 435

Cannizzo, J. K. 1998, ApJ, 494, 366

Cannizzo, J. K. 2000, ApJ, 534, L35

Chen, W., Shrader, C. R., \& Livio, M. 1997, ApJ, 491, 312

Chen, X., Abramowicz, M. A., Lasota, J.-P., Narayan, R., \& Yi, I. 1995, ApJ, 443, L61

Corbel, S., Fender, R. P., Tomsick, J. A., et al. 2004, ApJ, 617, 1272

de Kool, M., \& Wickramasinghe, D. 1999, MNRAS, 307, 449

Done, C., \& Gierliński, M. 2004, in Stellar-Mass, IntermediateMass, and Supermassive Black Holes, ed. K. Makishima, \& S. Mineshige, Progr. Theor. Phys. Suppl., 155, 9

Dubus, G., Hameury, J.-M., \& Lasota, J.-P. 2001, A\&A, 373, 251

Ebisawa, K., Ogawa, M., \& Aoki, T. 1994, PASJ, 46, 375

Esin, A. A., McClintock, J. E., \& Narayan, R. 1997, ApJ, 489, 865

Gilfanov, M., Churazov, E., \& Revnivtsev, M. 2000, MNRAS, 316, 923

Homan, J., \& Belloni, T. 2005 [arXiv: astro-ph/0412597]

Kalemci, E., Tomsick, J. A., Buxton, M. M., et al. 2005, ApJ, 622, 508

Kubota, A., \& Done, C. 2004, MNRAS, 353, 980

Ling, J. C., \& Wheaton, Wm. M. 2005, ApJ, 622, 492

Liu, B. F., Mineshige, S., Meyer, F., et al. 2002, ApJ, 575, 117

Liu, B. F., Meyer, F., \& Meyer-Hofmeister, E. 2004, A\&A, 421, 659

Maccarone, T. J., \& Coppi, P. S. 2003a, MNRAS, 338, 189

Maccarone, T. J., \& Coppi, P. S. 2003b, A\&A, 399, 1151

Maitra, D., \& Bailyn, C. D. 2004, ApJ, 608, 444

McClintock, J. E., \& Remillard, R. A. 2005, to appear in Compact Stellar X-ray Sources, ed. W. H. G. Lewin, \& M. van der Klis (Cambridge University Press) [arXiv: astro-ph 0306213]

Meyer-Hofmeister, E. 2004, A\&A, 423, 321

Meyer-Hofmeister, E., \& Meyer, F. 1999, A\&A, 348, 154

Meyer-Hofmeister, E., \& Meyer, F. 2001, A\&A, 380, 739

Meyer-Hofmeister, E., \& Meyer, F. 2003, A\&A, 402, 1013 
Meyer-Hofmeister, E., Liu, B. F., \& Meyer, F. 2005, A\&A, 432, 181 (MLM05)

Meyer, F., Liu, B. F., \& Meyer-Hofmeister, E. 2000, A\&A, 361, 175 Mineshighe, S., Liu, B. F., Meyer, F., et al. 1998, PASJ, 50, L5

Mitsuda, K., Inoue, H., Nakamura, N., et al. 1989, PASJ, 41, 97

Miyamoto, S., Kitamoto, S., Hayashida, K., et al. 1995, ApJ, 442, L13

Muno, M. P., Remillard, R. A., \& Chakrabarty, D. 2002, ApJ, 568, L35

Naik, S., Nagrawal, B. P., Rao, et al. 2000, A\&A, 354, 938

Narayan, R., Mahadevan, R., \& Quataert, E. 1998, in The Theory of Black Hole Accretion Discs, ed. M. A. Abramowicz et al. (Cambridge Univ. Press), 48

Narayan, R., \& McClintock, J. E. 2005, ApJ, 623, 1017

Olive, J.-F., Baret, D., \& Gierliński, M. 2003, ApJ, 583, 416

Park, S. Q., Miller, J. M., McClintock, J. E., et al. 2004, ApJ, 610, 378

Quataert, Q. E., Di Matteo, T., \& Narayan, R. 1999, ApJ, 525, L89
Rodriguez, J., Corbel, S., \& Tomsick, J. A. 2003, ApJ, 595, 1032

Rossi, S., Homan, J., Miller, J., et al. 2004, to appear in Proc. of the II BeppoSAX Meeting, ed. E. P. J. van den Heuvel, J. J. M. in 't Zand, \& R. A. M. J. Wijers, Nuclear Physics B Proc. Suppl., 132,416

Różańska, A., \& Czerny, B. 2000, A\&A, 360, 1170

Tanaka, Y., \& Shibazaki, N. 1996, ARA\&A, 34, 607

Wardziński, G., Zdziarski, A. A., \& Gierliński, M. 2002, MNRAS, 337, 829

Yuan, F., \& Narayan, R. 2004, ApJ, 612, 724

Zdziarski, A. A., Poutanen, J., \& Mikolajewska, J. 1998, MNRAS, 301,435

Zdziarski, A. A., \& Gierliński, M. 2004, in Stellar-Mass, IntermediateMass, and Supermassive Black Holes, ed. K. Makishima, \& S. Mineshige, Progr. Theor. Phys. Suppl. 155, 99

Życki, P. T., Done, C., \& Smith, D. A. 1998, ApJ, 496, L25 